\title{
Systematic review and meta-analysis on influence of human papillomavirus infection during pregnancy on premature rupture of membranes and premature delivery
}

\author{
Dongyan Wu ${ }^{1 \#}$, Lili Chen ${ }^{1 \#}$, Juan Zhen ${ }^{2}$, Xia Jin ${ }^{1}$ \\ ${ }^{1}$ Obstetrics and Gynecology Department, The Fourth Hospital of Shijiazhuang, Shijiazhuang, China; ${ }^{2}$ Department of Pathology, The Fourth \\ Hospital of Shijiazhuang, Shijiazhuang, China \\ Contributions: (I) Conception and design: D Wu, L Chen; (II) Administrative support: J Zhen; (III) Provision of study materials or patients: D Wu, L \\ Chen, X Jin; (IV) Collection and assembly of data: All authors; (V) Data analysis and interpretation: D Wu, J Zhen, X Jin; (VI) Manuscript writing: \\ All authors; (VII) Final approval of manuscript: All authors. \\ \#These authors contributed equally to this work. \\ Correspondence to: Dongyan Wu. Obstetrics and Gynecology Department, The Fourth Hospital of Shijiazhuang, Shijiazhuang Tanggubei Street No. \\ 16, Shijiazhuang, China. Email: wu13582105879@163.com.
}

\begin{abstract}
Background The increasing infection rate of human papillomavirus (HPV) has resulted in various complex pregnancy-related complications in recent years. HPV can directly pass through the placenta to cause intrauterine infection, leading to premature delivery or the premature rupture of membranes (PROM).

Methods: English databases were searched for randomized control trials (RCTs) on HPV infection and premature delivery and PROM, including PubMed, Medline, Embase, and Cochrane Central Register. The search time was from inception to March 1st 2021, with human papillomavirus, rupture, pregnancy, preterm birth, viral infection, and pregnancy complications as search terms. RevMan5.3 provided by the Cochrane Collaboration was used to perform bias risk assessment.
\end{abstract}

Results: A total of 7 studies were identified, involving 45,603 patients, including 22,799 cases in the control group, and 22,799 cases in an HPV infection group. The odds ratio (OR) and $95 \%$ confidence interval (95\% CI) were used to express the results. HPV infection increased the probability of premature delivery $(\mathrm{OR}=1.81,95 \% \mathrm{CI}: 1.25-2.62, \mathrm{Z}=3.16, \mathrm{P}=0.002)$ and $\mathrm{PROM}(\mathrm{OR}=1.74,95 \% \mathrm{CI}: 1.45-2.10, \mathrm{Z}=5.84$, $\mathrm{P}<0.00001)$. The $\mathrm{P}$ values were all less than 0.05 , and the difference was statistically significant.

Discussion: A total of 7 articles were included in this meta-analysis. HPV infection affects the physiology of pregnant women and may lead to PROM and premature delivery.

Keywords: Premature rupture of membranes (PROM); premature delivery; meta-analysis; human papilloma virus (HPV)

Submitted Aug 12, 2021. Accepted for publication Oct 14, 2021.

doi: 10.21037/apm-21-2497

View this article at: https://dx.doi.org/10.21037/apm-21-2497

\section{Introduction}

Human papillomavirus (HPV) is a small double-stranded closed circular DNA virus with high host-specific. It is named because some types of HPV can cause papillomas or warts in certain parts of the skin mucosa (1). More than $80 \%$ of men and women over 45 years old have a history of HPV infection, and women of young reproductive age are predominantly affected. Pregnant women are more susceptible to HPV infection due to their special endocrine and immune status, and it has been reported that the cervical HPV infection rate in this group is $82 \%$, which is significantly higher than the $10.4 \%$ in nonpregnant women (2). The HPV detection rate for pregnant women is $15.53 \%$, which is also higher than the $12.6 \%$ of 
non-pregnant women and confirms the susceptibility of pregnant women to HPV infection $(3,4)$. HPV infection during pregnancy leads to adverse pregnancy outcomes, such as miscarriage, premature delivery, premature rupture of membranes, preeclampsia and restriction of intrauterine growth and development of the fetus, which seriously affects the health of the newborn and prevents the smooth delivery of the newborn. This is a common concern of obstetricians and pregnant women (5).

In recent years, the pregnancy outcomes resulting from HPV infection have attracted much concern (6). The World Health Organization defines premature delivery as a delivery within 37 gestational weeks, and in developed countries, pregnant women usually have HPV tests at 22 or 24 weeks (7). However, in developing countries, the lower limit for premature babies is 28 weeks of gestation and a birth weight of $1,000 \mathrm{~g}$ (8). Factors that induce premature delivery include lower genital duct and urinary tract infections, intrauterine infections, uterine hyperdilatation, uterine malformations, placental and internal organ factors, diarrhea, and other pregnancy complications (9). $\mathrm{HPV}$ infection is an important factor in the occurrence of premature delivery and can lead to intrauterine infection (10). When a pregnant woman is infected with HPV, the vaginal environment becomes more susceptible to bacterial disease. Moreover, infection with pathogenic microorganisms in the reproductive duct is associated with premature rupture of the uterine membranes (11). Some scholars found that HPV infection has a great impact on pregnancy outcomes, such as immature delivery, immature membrane rupture, postpartum hemorrhage, puerperal infection, and fetal growth restriction $(12,13)$. Current thinking holds that highrisk HPV infection is closely associated with premature delivery, but the hypothesis has not been verified. Hence, a systematic review of existing studies is necessary to obtain more accurate conclusions to guide clinical practice (14).

The innovation of this paper lies in the fact that it is necessary to explore the correlation between high-risk HPV infection and adverse pregnancy outcomes. Many scholars at home and abroad have conducted a series of observational studies, but the results are not completely consistent, that is, there is controversy over whether there is correlation between the two (15). In this research, the correlation between premature rupture of membranes and premature delivery was analyzed by human papillomavirus infection during pregnancy (16). In this meta-analysis, 7 randomized control trials (RCTs) on the influence of $\mathrm{HPV}$ on the premature rupture of membranes (PROM) and premature delivery were included, for the purpose of establishing a theoretical basis for the prevention of PROM and premature delivery during pregnancy and improving the survival rate of pregnant women.

We present the following article in accordance with the PRISMA reporting checklist (available at https://dx.doi. org/10.21037/apm-21-2497).

\section{Methods}

\section{Literature retrieval}

The Medline, Embase, and Cochrane Central Register of Controlled Trials databases were searched for RCTs on the influence of HPV on PROM and premature delivery published from January $1^{\text {st }}, 2001$ to March $1^{\text {st }}, 2021$, with human papillomavirus, PROM, pregnancy, premature birth, viral infection, pregnancy complications, gestational diabetes mellitus, gestational hypertensive disease, chorioamnionitis, cervical dysplasia, preeclampsia, and spontaneous abortion as search terms. Additionally, the symptoms and treatment of other complications during pregnancy were sought.

\section{Inclusion and exclusion criteria}

The following inclusion criteria applied: (I) those diagnosed with HPV infection within 3 years before pregnancy to 6 months after delivery; (II) with premature delivery and PROM; (III) RCT; and (IV) published literature.

The exclusion criteria were: (I) Chinese literature; (II) meta-analyses not relevant to the topic; or (III) literature with unclear results and incomplete data.

\section{Literature screening}

Initially, some references were eliminated after reading the titles and abstracts. Then, a second screening was performed according to the inclusion criteria and exclusion criteria, and references were traced using a search engine. Finally, a third screening was conducted by reading the full text of the included literature.

\section{Quality assessment}

The Newcastle-Ottawa Scale (NOS) was used to evaluate the quality of the literature according to seven criteria: (I) whether a randomized method was used; (II) whether 
allocation concealment was used; (III) whether the subject and the operator were blinded; (IV) whether the assessor was blinded; (V) whether the results data was complete; (VI) whether the results were reliable; and (VII) other biases. Each item accounted for 1 point, and the total score ranged from 0-7 points. A score of 4-7 points was considered high quality research, and 0-3 was considered low quality research.

\section{Data extraction}

Two experts used unified Microsoft Excel to collate data independently, and inconsistency was resolved by discussions or inviting another expert to arbitrate. The following data were collated: (I) research title, first author's name, publication year, and publication name; (II) general information of the research object: region, sample size, gender ratio, and age distribution; (III) observation indicators; and (IV) intervention methods in the control group and experimental group.

\section{Risk of bias and quality assessment of articles}

The Cochrane randomized trial risk assessment tool was applied to evaluate the quality of the included articles. The evaluation included the following six items: (I) the random sequence generation method; (II) whether there was bias in the allocation process; (III) whether the blind research was adopted; (IV) whether the results data was complete; (V) whether there was selective reporting of the research results; and (VI) other deviations.

\section{Sensitivity analysis}

Sensitivity analysis was used to evaluate whether the results of meta-analysis were stable and reliable, specifically by excluding some controversial studies, low-quality studies or analyzing the same group of data using statistical methods/effect models at different points to observe the changes in meta-analysis results. If the sensitivity analysis does not substantially change the results, the results are reliable. On the contrary, it is suggested that great caution should be exercised in interpreting results and drawing conclusions.

\section{Statistics analysis}

RevMan 5.3 software was used to analyze the risk bias of the included literature. The odds ratio (OR) was used as the effect size and $95 \%$ confidence interval (CI) was used to express the results. First, a heterogeneity test was perfumed on the included studies, with $\alpha=0.1$ as the cut-off value. If there was no heterogeneity between the studies $\left(\mathrm{P}>0.1, \mathrm{I}^{2}<50 \%\right)$, the fixed-effects model was selected for meta-analysis, otherwise, subgroup analysis was then performed. $\mathrm{P}<0.05$ was the threshold for significance. When a single risk factor analysis was included in more than 7 articles, a funnel chart was used to analyze the publication bias of the risk factor. RevMan5.3 software was used to analyze publication bias by Begg's and Egger's test methods and if $\mathrm{P}>0.05$, it was considered that there was no publication bias.

\section{Results}

\section{Literature retrieval results}

By searching keywords, 1,969 articles were initially obtained. After reading the titles of the above-mentioned articles, and excluding those that did not meet the requirements, 544 articles were used for the next analysis. After browsing the abstracts of the literature roughly, 76 articles were used for the next analysis. The literature was screened according to the inclusion and exclusion criteria, and 31 articles were used for the next analysis. After downloading and reading the documents, a total of 7 documents were finally used for meta-analysis (17-23). Figure 1 depicts the flowchart of literature retrieval, and Table 1 shows the basic information of the included studies. The included studies compared the treatment effects of the two methods according to the main methods used, and the methodological quality of each research in the intervention results was medium or above, at the same level of literature quality, and there was no methodological heterogeneity. Therefore, this systematic review did not conduct sensitivity analysis.

\section{Risk of bias of the included literature}

The Cochrane Handbook version 5.3 was used to evaluate the bias risk of the 7 articles included in this meta-analysis. As shown in Figure 2 and Figure 3, Review Manager 5.3 software was used to output the risk bias maps.

\section{Meta-analysis of HPV infection and PROM}

As shown in Figure 4 and Figure 5, there were 2 articles 


\section{Identification of studies via databases and registers}

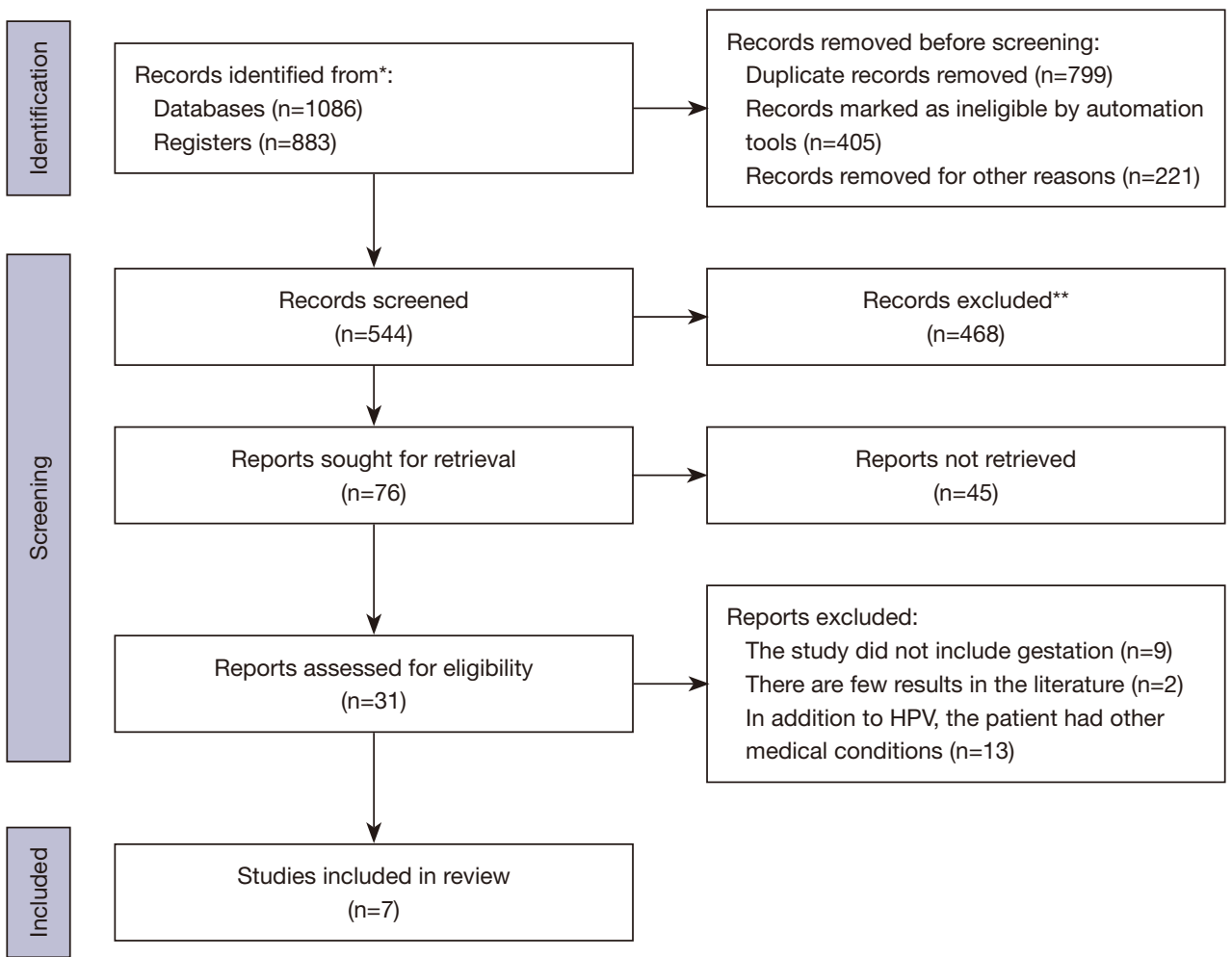

Figure 1 Flowchart of literature retrieval. *, consider, if feasible to do so, reporting the number of records identified from each database or register searched (rather than the total number across all databases/registers); **, if automation tools were used, indicate how many records were excluded by a human and how many were excluded by automation tools.

Table 1 Basic information of the included literature

\begin{tabular}{|c|c|c|c|c|}
\hline First author & Publication year & Consequences & Age/year & Research duration \\
\hline Gomez (18) & 2008 & PROM & Not clear & 36 weeks of pregnancy \\
\hline Cho (19) & 2013 & $\begin{array}{l}\text { Premature delivery, PROM, preeclampsia, and } \\
\text { gestational diabetes (GDM) }\end{array}$ & Not clear & End of pregnancy \\
\hline McDonnold (20) & 2014 & Preeclampsia, premature delivery & Not clear & $\begin{array}{l}\text { Prenatal care to the end of } \\
\text { pregnancy }\end{array}$ \\
\hline Slatter (21) & 2015 & Premature delivery, preeclampsia, and diabetes & Not clear & Whole pregnancy \\
\hline Subramaniam (22) & 2016 & $\begin{array}{l}\text { Hypertension during pregnancy, preeclampsia or } \\
\text { eclampsia, premature delivery }\end{array}$ & Not clear & Whole pregnancy \\
\hline Ambühl (23) & 2017 & Premature delivery or spontaneous abortion & Over 18 & 8-22 weeks \\
\hline
\end{tabular}

PROM, premature rupture of membranes. 


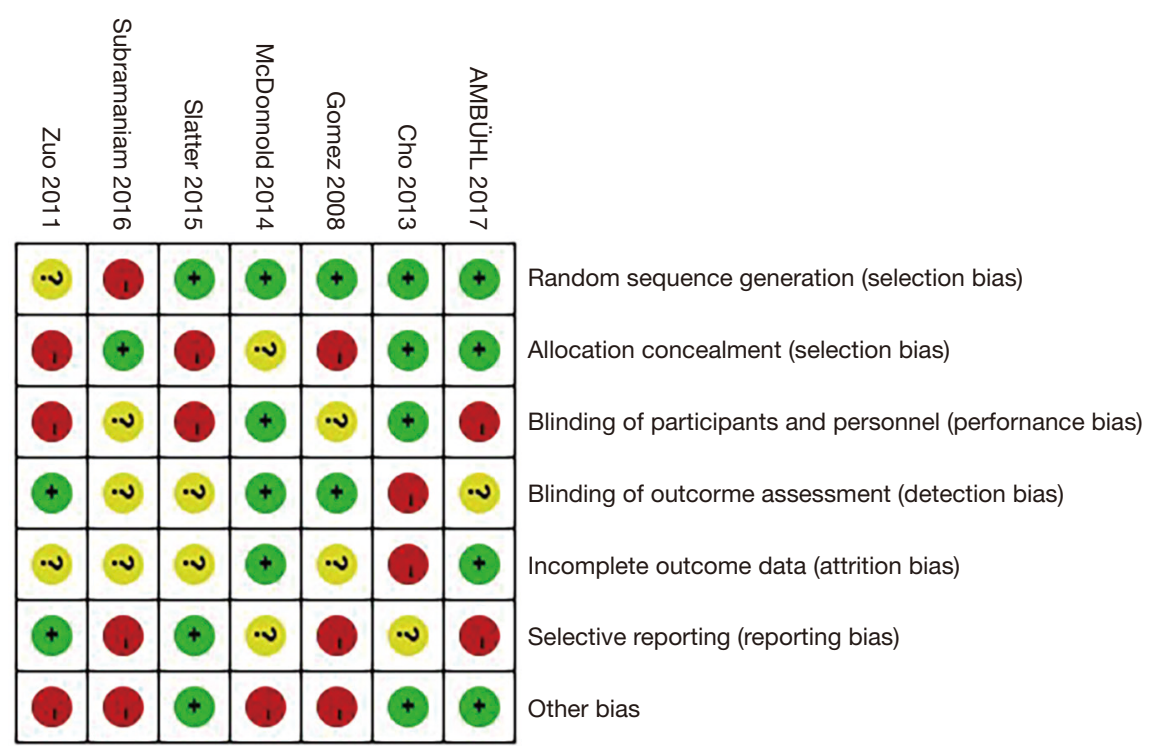

Figure 2 Bias risk assessment results of included literature.

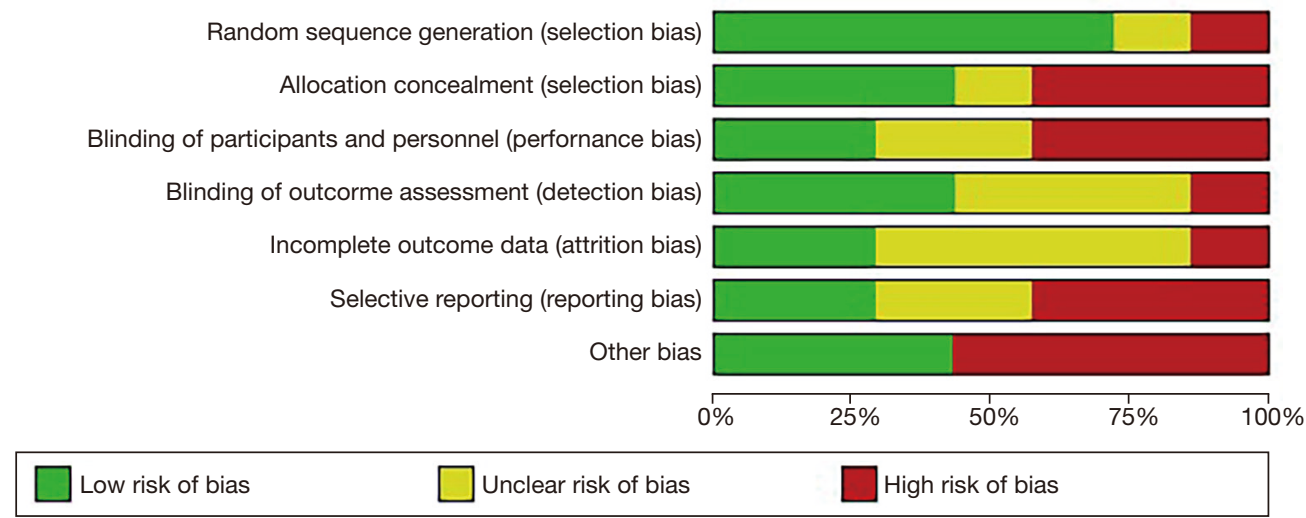

Figure 3 Bar chart showing the bias risk assessment results of the included literature.

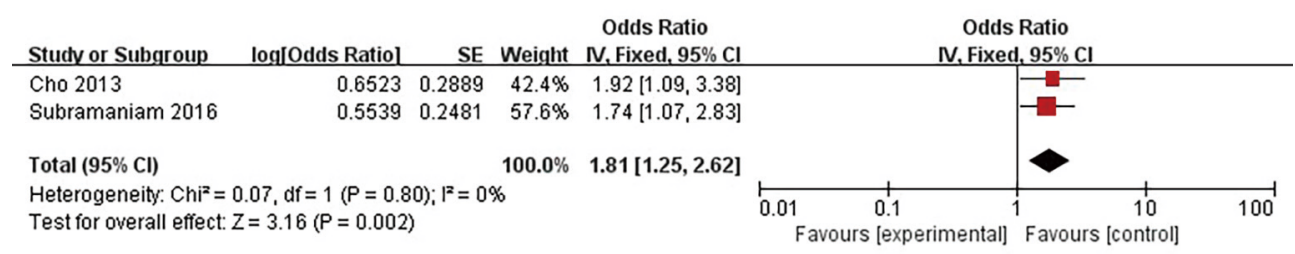

Figure 4 Forest plot of premature rupture of membranes in two groups.

with premature rupture of membranes as the outcome index, and both of which were cohort studies. Meta-analysis of each included data showed that there was no statistical heterogeneity $\left(\mathrm{I}^{2}=0 \%, \mathrm{P}=0.80\right)$, and a fixed effect model
(FEM) was used for analysis. The results of the combined effect model showed that pregnant women with high-risk HPV infection had a higher incidence of premature rupture of membranes than the negative control group, and the 


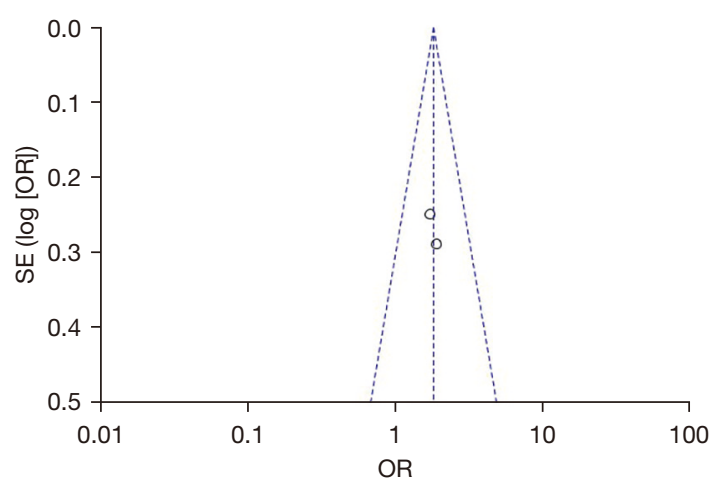

Figure 5 Funnel diagram of premature rupture of membranes. SE, standard error; OR, odds ratio.

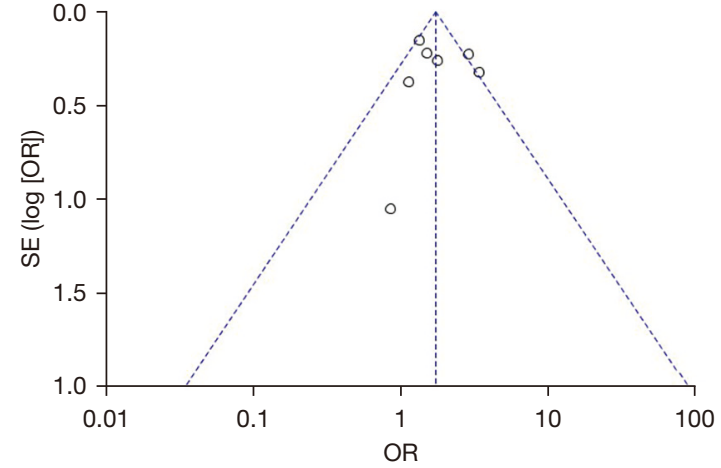

Figure 7 Funnel chart of premature delivery in two groups. SE, standard error; OR, odds ratio.

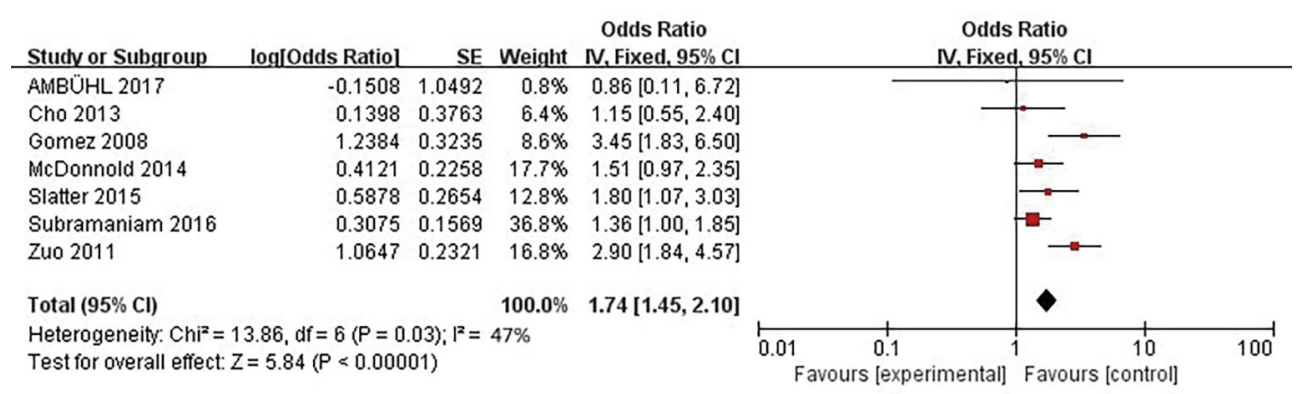

Figure 6 Forest plot of premature delivery in two groups.

difference between the two groups was statistically obvious ( $\mathrm{OR}=1.81,95 \% \mathrm{CI}: 1.25-2.62, \mathrm{Z}=3.16, \mathrm{P}=0.002)$. It can be concluded that women with high-risk HPV infection were more prone to premature rupture of membranes.

\section{Meta-analysis of HPV infection and premature delivery}

As shown in Figure 6 and Figure 7, there were 7 articles with preterm birth as the main outcome indicator, all of which were cohort studies. Analysis of the included data showed that there was no statistical heterogeneity $\left(\mathrm{I}^{2}=47 \%, \mathrm{P}=0.03\right)$, and the FEM was used for effect size combination. The results showed that the high-risk HPV infection positive group had a higher incidence of preterm birth in the higherrisk HPV negative group, and the difference between the two groups was statistically significant $(\mathrm{OR}=1.74,95 \%$ CI: $1.45-2.10, Z=5.84, P<0.00001)$, so women with highrisk HPV infection were more likely to have premature delivery. In the sensitivity analysis of the combined results, none of the articles violated the total combined effect size. In other words, the results obtained were relatively stable.
Therefore, a definite conclusion can be drawn: women with high-risk HPV infection were more likely to have premature delivery, so there was no publication bias in the symmetry of the funnel graph.

\section{Discussion}

This meta-analysis included a total of 7 studies (17-23), one of which was of high-quality and three of which were low-quality. Three studies reported basic data such as age, disease type, and disease stage, but there were no statistically significant differences between the experimental group and the control group $(\mathrm{P}>0.05)$. One article (18) introduced a random number table, and another (19) mentioned random grouping according to the pregnancy time. three studies $(18,20,21)$ introduced random methods, but did not mention the measurement blindness, nor did they report the number lost to follow up. The limitations of interventional measures indicated there was measurement bias, and improvements in methodology appear to be required to improve the reliability of studies in the future. Studies have shown 
that HPV infection can cause the human body to produce terminal Phospholipids A2 on the fetal cell membrane, and then produce a series of hormones and enzymes, such as tyrosinase protein. These enzymes decompose the fetal membrane matrix and collagen, and eventually lead to the early rupture of the maternal fetal membrane (24). At the same time, the membrane tissue of the infected fetus activates various physiological reactions, synthesizes a large amount of prostaglandin, and stimulates contraction of the uterus. Pathogenic microorganisms invading the human body increase the activity of proteases and reduce the secretion of immune body fluids from the cervix. The premature rupture of the fetal membrane will not only lead to premature delivery of the fetus, but the infection itself will also increase the risk of premature delivery, because inflammatory factors increase, and the neck tube detaches, causing premature delivery. However, whether HPV infection can cause premature delivery remains to be verified, and results to date have varied. In examining 322 cases infected with HPV as research subjects, Lawton et al. (25) found HPV infection increased the risk of early membrane rupture and premature delivery. However, Xiong et al. (26) found HPV-positive women did not have an increased risk of premature birth compared with HPVnegative women, and that HPV infection did increase the risk of PROM. In another meta-analysis, Bonde et al. found $\mathrm{HPV}$ infection increased the risk of premature delivery, although there was no discussion regarding PROM in their study (27).

Meta-analysis of the included data in this study showed that pregnant women with high-risk HPV infection had a higher incidence of premature rupture of membranes than those in the negative control group, and women with highrisk HPV infection were more likely to have premature rupture of membranes. In addition, the results of this study showed that the high-risk HPV infection positive group had a higher incidence of preterm birth in the higher-risk HPV negative group, and the difference between the two groups was statistically significant. Therefore, it is said that women with high-risk HPV infection are more likely to have preterm birth. In addition, the Begg's and Egger's methods were applied to test publication bias, and the $\mathrm{P}$ values were both greater than 0.05 , showing that there was no publication bias. HPV vaccine acts as primary prevention of cervical cancer for the receptor protection. With the deepening of clinical studies on the effectiveness of HPV vaccine in preventing HPV virus infection, universal prophylactic vaccination of HPV vaccine can be widely used to reduce cervical cancer, precancerous lesions, and adverse pregnancy outcomes. Recommended for all women user papilloma virus (HPV) vaccine, because of HPV vaccine clinical trials do not include pregnant women, known HPV vaccination during pregnancy is not recommended, but there are still some people choose to vaccination during pregnancy, especially during the first three months of pregnancy, the one who was unconscious or unable to identify pregnant will inadvertently vaccination. However, the safety of the HPV vaccine in pregnancy needs to be further studied. HPV infection, the second most sexually transmitted disease after gonorrhea, is not only linked to cervical cancer, but also increases the chances of adverse pregnancy outcomes. It hopes to include HPV screening as a physical examination project like gynecological ultrasound, especially in pre-marital examination and prepregnancy examination, as early as possible, to reduce the adverse effects of HPV infection.

This meta-analysis included several foreign articles studying various risk factors. However, the treatment time of the 7 included studies is contradictory, which may reduce the power of the results, and there is no report on the randomization method. Therefore, we encourage future researchers in this field to further improve the experimental plan, standardize the specific time, methods, and drugs of periodic interventions, and select high-quality, large-scale samples to obtain more reliable evidence the reliability of their results.

\section{Conclusions}

This meta-analysis found that HPV infection can affect the physiological conditions of pregnant women during pregnancy, leading to PROM and premature delivery. The meta-analysis results were basically stable, but there are still the following limitations. Due to differences in retrieval mechanisms, the retrieval may be incomplete. There are also differences in risk factors in distinct references, which may affect the reliability of the results. In addition, the studies included are all publicly published literature, and there may be potential publication bias. We recommend the conduct of more high-quality, multi-center, largesample original research studies to provide a more reliable theoretical basis for the prevention of complications during pregnancy and improve the survival rate of pregnant women and fetuses. 


\section{Acknowledgments}

Funding: Key Science and Technology Research Plan of Hebei Provincial Department of Health (20171023).

\section{Footnote}

Reporting Checklist: The authors have completed the PRISMA reporting checklist. Available at https://dx.doi. org/10.21037/apm-21-2497

Conflicts of Interest: All authors have completed the ICMJE uniform disclosure form (available at https://dx.doi. org/10.21037/apm-21-2497). The authors have no conflicts of interest to declare.

Ethical Statement: The authors are accountable for all aspects of the work in ensuring that questions related to the accuracy or integrity of any part of the work are appropriately investigated and resolved.

Open Access Statement: This is an Open Access article distributed in accordance with the Creative Commons Attribution-NonCommercial-NoDerivs 4.0 International License (CC BY-NC-ND 4.0), which permits the noncommercial replication and distribution of the article with the strict proviso that no changes or edits are made and the original work is properly cited (including links to both the formal publication through the relevant DOI and the license). See: https://creativecommons.org/licenses/by-nc-nd/4.0/.

\section{References}

1. Bonde U, Joergensen JS, Lamont RF, et al. Is HPV vaccination in pregnancy safe? Hum Vaccin Immunother 2016;12:1960-4.

2. Faber MT, Duun-Henriksen AK, Dehlendorff C, et al. Adverse pregnancy outcomes and infant mortality after quadrivalent HPV vaccination during pregnancy. Vaccine 2019;37:265-71.

3. Bober L, Guzowski G, Moczulska H, et al. Influence of human Papilloma Virus (hPV) infection on early pregnancy. Ginekol Pol 2019;90:72-5.

4. López-Fauqued M, Zima J, Angelo MG, et al. Results on exposure during pregnancy from a pregnancy registry for AS04-HPV-16/18 vaccine. Vaccine 2017;35:5325-30.

5. Hornychova H, Kacerovsky M, Musilova I, et al. Cervical human papillomavirus infection in women with preterm prelabor rupture of membranes. PLoS One 2018; 13:e207896.

6. Wiik J, Nilsson S, Kärrberg C, et al. Associations of treated and untreated human papillomavirus infection with preterm delivery and neonatal mortality: A Swedish population-based study. PLoS Med 2021;18:e1003641.

7. Pandey D, Solleti V, Jain G, et al. Human Papillomavirus (HPV) Infection in Early Pregnancy: Prevalence and Implications. Infect Dis Obstet Gynecol 2019;2019:4376902.

8. Jar-Allah T, Kärrberg C, Wiik J, et al. Abnormal cervical cytology is associated with preterm delivery: A population based study. Acta Obstet Gynecol Scand 2019;98:777-86.

9. van de Laar RLO, Hofhuis W, Duijnhoven RG, et al. Adjuvant VACcination against HPV in surgical treatment of Cervical Intra-epithelial Neoplasia (VACCIN study) a study protocol for a randomised controlled trial. BMC Cancer 2020;20:539.

10. Schwarz TF, Huang LM, Valencia A, et al. A tenyear study of immunogenicity and safety of the AS04HPV-16/18 vaccine in adolescent girls aged 10-14 years. Hum Vaccin Immunother 2019;15:1970-9.

11. Meller CH, Carducci ME, Ceriani Cernadas JM, et al. Preterm premature rupture of membranes. Arch Argent Pediatr 2018;116:e575-81.

12. Koizumi K, Fujioka T, Yasuoka T, et al. Clinical investigation of the safety and efficacy of a cervical intraepithelial neoplasia treatment using a hyperthermia device that uses heat induced by alternating magnetic fields. Mol Clin Oncol 2016;5:310-6.

13. Castle PE, Aslam S, Behrens C. Cervical Precancer and Cancer Risk by Human Papillomavirus Status and Cytologic Interpretation: Implications for Risk-Based Management. Cancer Epidemiol Biomarkers Prev 2016;25:1595-9.

14. Arbyn M, Redman CWE, Verdoodt F, et al. Incomplete excision of cervical precancer as a predictor of treatment failure: a systematic review and meta-analysis. Lancet Oncol 2017;18:1665-79.

15. Hetea A, Cosconel C, Stanescu AAM, et al. Alcohol and Psychoactive Drugs in Pregnancy. Maedica (Bucur) 2019;14:397-401.

16. Huang QT, Zhong M, Gao YF, et al. Can HPV vaccine have other health benefits more than cancer prevention? A systematic review of association between cervical HPV infection and preterm birth. J Clin Virol 2014;61:321-8.

17. Zuo Z, Goel S, Carter JE. Association of cervical cytology and HPV DNA status during pregnancy with placental 
abnormalities and preterm birth. Am J Clin Pathol 2011;136:260-5.

18. Gomez LM, Ma Y, Ho C, et al. Placental infection with human papillomavirus is associated with spontaneous preterm delivery. Hum Reprod 2008;23:709-15.

19. Cho G, Min KJ, Hong HR, et al. High-risk human papillomavirus infection is associated with premature rupture of membranes. BMC Pregnancy Childbirth 2013;13:173.

20. McDonnold M, Dunn H, Hester A, et al. High risk human papillomavirus at entry to prenatal care and risk of preeclampsia. Am J Obstet Gynecol 2014;210:138.e1-5.

21. Slatter TL, Hung NG, Clow WM, et al. A clinicopathological study of episomal papillomavirus infection of the human placenta and pregnancy complications. Mod Pathol 2015;28:1369-82.

22. Subramaniam A, Lees BF, Becker DA, et al. Evaluation of Human Papillomavirus as a Risk Factor for Preterm Birth or Pregnancy-Related Hypertension. Obstet Gynecol 2016;127:233-40.

23. Ambühl LMM, Leonhard AK, Widen Zakhary C, et al. Human papillomavirus infects placental trophoblast and

Cite this article as: $\mathrm{Wu} \mathrm{D}$, Chen L, Zhen J, Jin X. Systematic review and meta-analysis on influence of human papillomavirus infection during pregnancy on premature rupture of membranes and premature delivery. Ann Palliat Med 2021;10(10):1073510743. doi: 10.21037/apm-21-2497
Hofbauer cells, but appears not to play a causal role in miscarriage and preterm labor. Acta Obstet Gynecol Scand 2017;96:1188-96.

24. Scheller NM, Pasternak B, Mølgaard-Nielsen D, et al. Quadrivalent HPV Vaccination and the Risk of Adverse Pregnancy Outcomes. N Engl J Med 2017;376:1223-33.

25. Lawton B, Howe AS, Turner N, et al. RETRACTED: Association of prior HPV vaccination with reduced preterm birth: A population based study. Vaccine 2018;36:134-40.

26. Xiong YQ, Mo Y, Luo QM, et al. The Risk of Human Papillomavirus Infection for Spontaneous Abortion, Spontaneous Preterm Birth, and Pregnancy Rate of Assisted Reproductive Technologies: A Systematic Review and Meta-Analysis. Gynecol Obstet Invest 2018;83:417-27.

27. Bonde U, Joergensen JS, Mogensen O, et al. The potential role of $\mathrm{HPV}$ vaccination in the prevention of infectious complications of pregnancy. Expert Rev Vaccines 2014;13:1307-16.

(English Language Editor: B. Draper) 